\title{
VanderLugt Correlator and Neural Networks
}

Eung Gi Paek, John .R. Wullert II, A. Von Lehmen, J.S. Patel, A. Scherer, J. Harbison, H.J. Yu, R. Martin

Belicore

331 Newman Springs Road

Red Bank, New Jersey 07701

\section{ABSTRACT}

The VanderLugt correlator, which was developed 25 years ago for the purpose of optical pattern recognition, still plays an important role in implementing recent neural network models. Several implementations of neural network models by using the VanderLugt correlator will be described. Furthermore, by using the recently developed surface emitting micro-laser diode array, the systems can be made compact and robust, still preserving the power of coherent optics.

\section{INTRODUCTION}

In recent years there has been interest in implementations of neural networks [1-3] in the hope of developing supercomputers to solve computationally massive problems such as pattern recognition by emulating the human brain. The features of neural computers can be described as follows: The algorithms attempt to emulate real neural networks. However, most recent neural network models are based on optimization principles. Although each computing element of neural nets is simple, parallel and analog processing through huge number of interconnections between input and output neurons gives the powerful computation capability. Therefore hardware implementation is important. Finally, neural computers are not intended for all-purpose computation. They are aimed at special purpose computations such as robust content addressable memories, speech or pattern recognition or learning that digital computers cannot perform properly.

Now we will describe about why optics, especially coherent optics, is powerful in implementing neural network models. The advantages of optics can be understood by the following two examples : Fourier optics [4] and a coherent optical correlator developed by VanderLugt[5].

To obtain the Fourier transform of a 20 (two-dimensional) image with a digital computer would require a large computational load. However, optics can do this job extremely well. By simply illuminating the input with a laser beam, the Fourier spectrum appears at the focal plane of a lens. By using such a simple lens, a continuous Fourier spectrum can be obtained at the speed of light. The computational power this simple lens can achieve is equivalent to $10^{21}$ multiplications $/ \mathrm{sec}$. The way optics performs such a massive computation is quite different from digital computers. It relies on a parallel and analogue processing through huge number of interconnections between the input and output, exactly as in neural computers.

Next, optics has a very powerful tool developed for pattern recognition purposes which can be directly used for neura network implementations. By using a simple holographic procedure developed by VanderLugt[5], all the targets on a paqe can be simultaneously recognized. This is perhaps the most powerful computer for pattern recognition. The computation is done in a parallel and analogue fashion as in neural computers, at the speed of light.

Therefore, optics is powerful especially for 2D pattern recognition because the operation is fully parallel along both $x$ and $y$ directions, and analog. Moreover, optics has huge number of available neurons, typically a million. Such a huge number of neurons in one layer can be connected into those in another layer through free space along the third dimension, eliminating the need for physical point-to-point connections. Finally, the wave nature of optics gives certain powerfu computations, such as Fourler transform, convolution, correlation and so on, which are crucial in neural network implementations. The simplicity of optics can be appreciated by considering that most computations required in neural network implementations can be achieved using a simple two-lens system.

In the following, several experimental demonstrations of neural networks using coherent optics will be demonstrated: An associative memory for word-break recognition and a learning machine for multicategory classification. Finally, a compact and robust coherent optical processor by using a recently developed SELDA (Surface Emitting micro-Laser Diode Array) will be described. Although the applications are different, all these implementations are based on the VanderLugt correlator.

\section{HOLOGRAPHIC ASSOCIATIVE MEMORY FOR WORD-BREAK RECOGNITION}

Since Hopfield[1] presented his model on a content addressable memory, many optical systems have been successfully demonstrated[6-18]. These are described in a review article by Owechko[19].

Recently, Hopfield and Tank[20-21] raised a new type of pattern recognition problem, known as the "word-break " problem, illustrated in the following sentence :

\section{TEXTREEDINGWITHAUTWORDBRECKSMAY BEKAMEDIFFICOLT}

Without spaces between words, it is difficult to decipher the text. The task becomes even more difficult if the continuous word stream contains errors. Deciphering the word stream requires a system which can recognize words each of which is embedded in a continuous stream of other letters, correct errors in the words, and insert spaces between them. The system must be shift invariant so that an input word can be recognized in any position. Since many words appear at the input window 
simultaneously, the system should also be able to process multiple inputs without crosstalk. Moreover, the system should be able to insert word-breaks at suitable positions between words.

By modifying a Hopfield-style holographic associative memory[17] to include the various features described above, a holographic word-break recognizer has been demonstrated $[22,23]$. As shown in Figure 1, memory words are

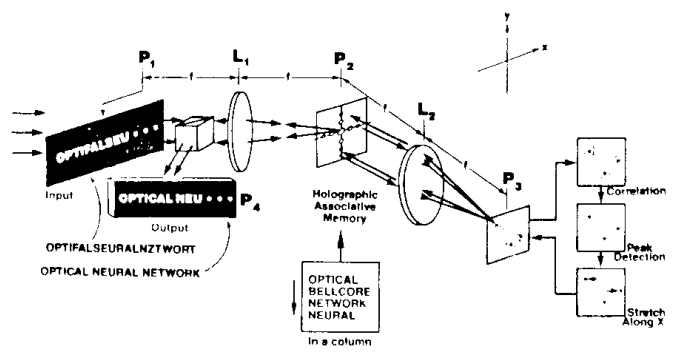

Fig. 1 Schematic diagram of the holographic word-break recognition system

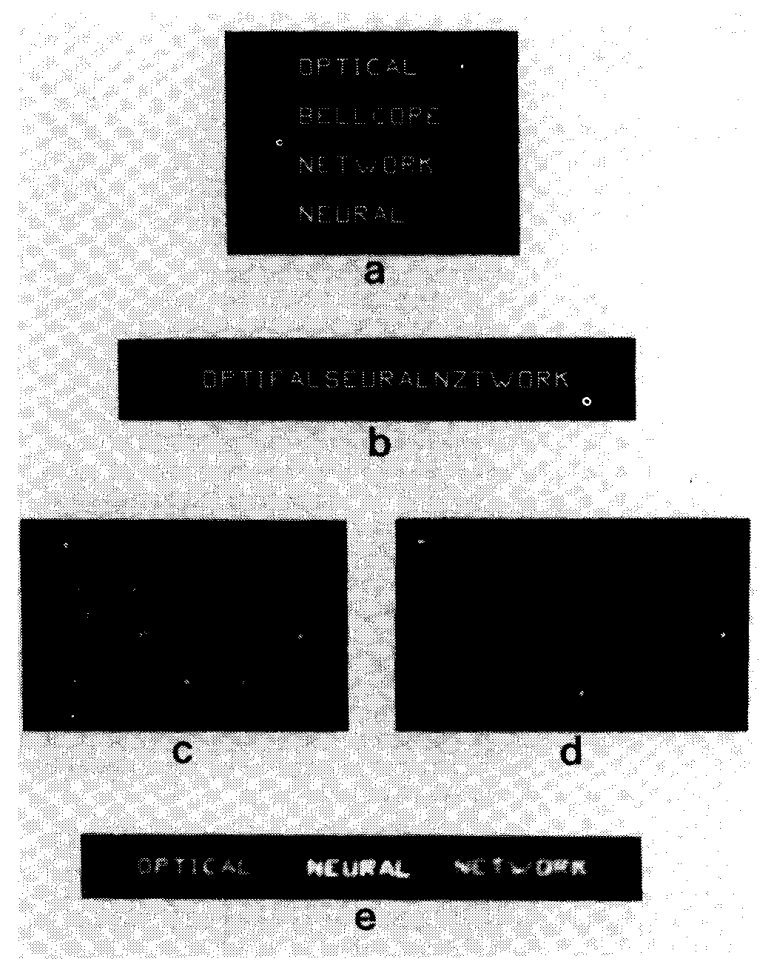

Fig. 2 (a) The four patterns stored in the holographic memory; (b) The input to be read; (c) correlation output ; (d) the thresholded version of the correlation output which is anamorphically magnified along the $x$ (word) direction ; and (e) the associative recalled output with spaces between words and error corrections. stored in a conventional Fourier transform hologram. The recognition part of the system consists mainly of a holographic VanderLugt correlator. For word-break recognition, the input word stream is presented at the input plane of the system. Autocorrelation peaks that appear at places where there is a match between the input and memory are detected and the separation between the peaks is magnified along the word direction. This stretched correlation output is reflected back to illuminate the hologram and reconstruct the whole memory at the output plane. The output through a window which is situated at the origin of the output plane is the desirable readable text. Figure 2 shows the experimental results. The four words used as the memories in this experiment are shown in Figure 2(a). The concatenated input with errors is shown in (b). The correlation output is shown in (c). The sharp autocorrelation peaks appear at the corresponding positions of the input words and memories. Also sidelobes appear over the whole correlation plane. Figure 2(d) shows the thresholded and anamorphically magnified version of the correlation output. The final output from the system is shown in Fig. 2(e). Compared with the initial input in (b), spaces are inserted between words and all the errors in the input are corrected.

\section{HOLOGRAPHIC LEARNING MACHINE FOR MULTICATEGORY CLASSIFICATION}

The recent increased activity in the area of neural networks is mainly due to the potential of neural computers to mimic the computational ability of humans, in particular the ability to learn. While the ordinary computer requires precise rules to perform a given task, the neural computer has the ability to learn its own rules from examples presented to it. This gives the neural computer the potentiai to solve problems not possible by artificial rule-based systems.

Most of the proposed[24-37] optical learning machines are based on the classical holographic setup with a photorefractive crystal as a medium to form variable interconnections between inputs and outputs. However in spite of the number of proposed systems and the current enthusiastic interest in implementing learning machines, the surprisingly few systems [34-37] that have been demonstrated so far can only classify inputs into two categories. A multicategory classifier[38] with many output neurons would be an important advance because it can classify inputs into many classes, making it possible to solve a greater range of problems than a twocategory classifier which is only capable of making 'yes / no' decisions.

We have recently demonstrated an optical learning machine that can function as a multicategory classifier[39] as shown in Fig. 3. The system is capable of classifying inputs into $2^{10}=1024$ possible output states. We achieve this performance by using bipolar interconnections and outputs, which allows us to implement the perceptron[2] learning algorithm, which in turn allows solution of problems not possible by unipolar representation

The system was tested to solve a small scale real-world problem of character recognition. Each of the input training patterns was loaded onto a two-dimensional spatial light modulator and focused onto a photorefractive crystal. The light diffracted from the crystal was collected and focused onto a detector array, which consists of 20 elements to represent the positive and negative values for ten outputs. The outputs from each pair of detectors were compared and the difference was thresholded as required for the perceptron algorithm. The error signals were generated by comparing the output with the 
desired target signals and were loaded onto a 20-element liquid crystal modulator (LCM), again to represent 10 bipolar error values.

Five standard capital letters were used as training patterns. Fig. 4 shows the learning curve (number of errors vs. Iteration) for the training sets. As shown in the Figure, the system successfully converged to the no error state after 10 iterations, even though the training sets are far from being orthogonal. The ultimate capacity of this system has not yet been investigated.

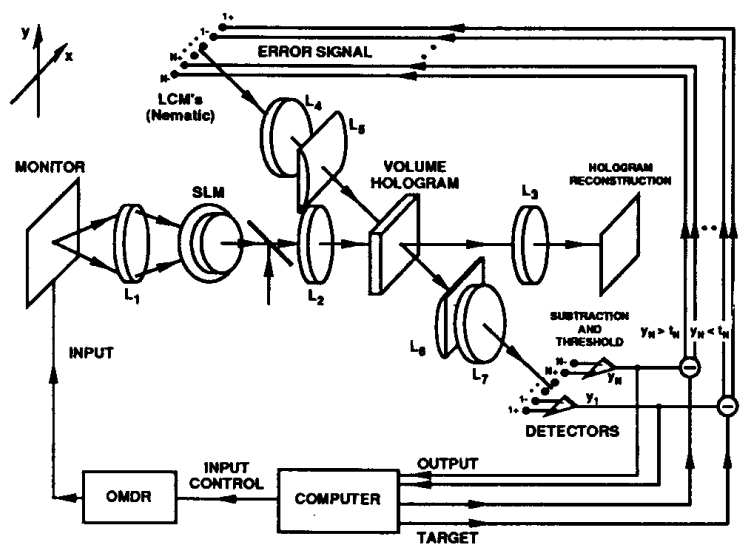

Fig. 3 Schematic of the holographic learning machine for multicategory classification.

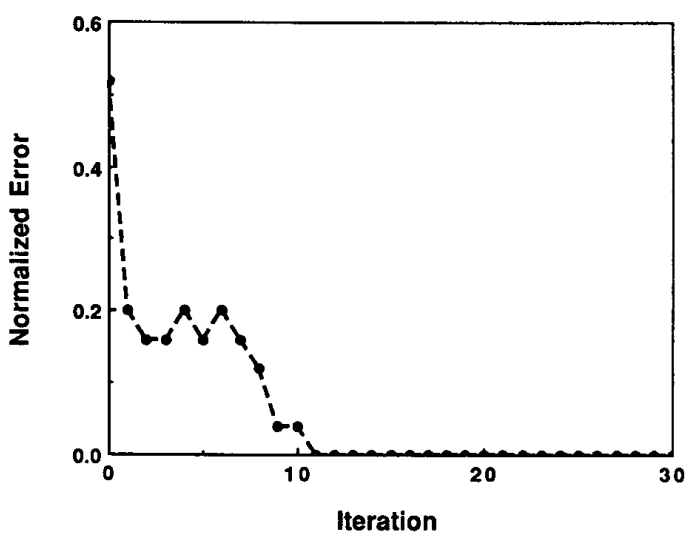

Fig. 4 Experimental learning curves for five training patterns ( $A$ $B, C, D$ and E).

\section{A COMPACT AND ULTRA-FAST HOLOGRAPHIC MEMORY USING A MICRO-LASER ARRAY}

As demonstrated in the two holographic implementations of neural network models, the VanderLugt correlator gives powerful computation and matches with neural networks very well. However, the problem is it requires a bulky laser and a heavy optical table and the alignment is critical. Therefore, it is difficult to cascade the system, which is required in the implementations of multi-layer neural networks[3]. The question is how to make the system compact and less critical, still preserving the computational power of coherent optics.

Recently, surface emitting micro-laser diode arrays (SELDA's) have been reported[40]. Figure 5 shows an SEM micrograph of the SELDA. The SELDA's have many features that make them highly desirable for use in holographic memory systems. The individual lasers can be as small as a few microns, allowing over

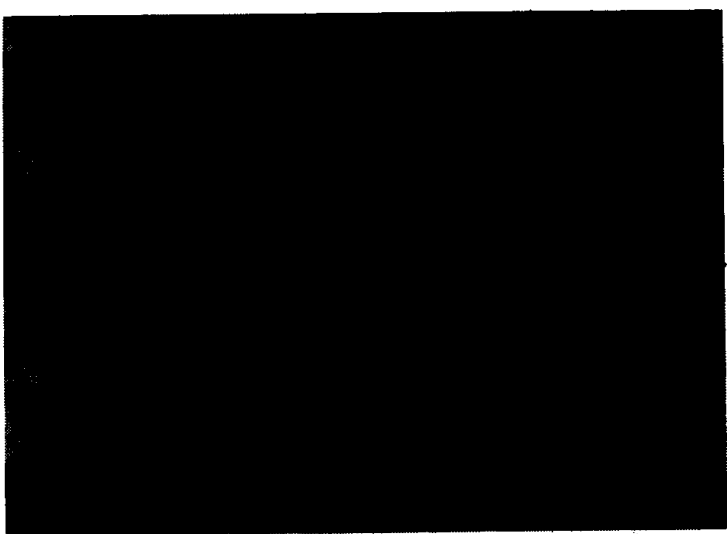

Fig. 5 SEM micrograph of the surface emitting micro-laser array.

one million microlasers on a $1 \mathrm{~cm}^{2}$ chip. The wavelength of the light from the laser is $960 \mathrm{~nm}$ with the linewidth of around $0.1 \mathrm{~nm}$. This gives a coherence length of several $\mathrm{cm}$ and the spectral resolution of about 10,000 . Therefore, a hologram of a high resolution image can be recorded and reconstructed by using an SEL. The threshold current of the laser is low, around $1 \mathrm{~mA}$ for lasers less than $5 \mathrm{~mm}$ in diameter, keeping device power requirements down. This low threshold is due to the high reflectivity of the DBR (distributed Bragg reflector) mirror structures, the use of quantum well structures in the active region and the small volume of the active layer. These enable effective photon and charge carrier confinement within the active layer. The output optical power from each laser can be more than $1 \mathrm{~mW}$. Therefore the light from each laser is bright enough to reconstruct a hologram which can be detected by a normal 2D Image sensor. The lasers exhlbit high switching speeds (100 psec) [40] allowing fast optical access and providing very high contrast since an OFF laser generates no light.

The issue here is how to utilize the novel device to make a coherent optical neuro-processor simple and compact. As can be seen in Figure 1, normal associative memories consist of two parts: recognition and readout, where the latter can be considered a holographic memory readout. For convenience of description, readout part will be described first.

Figure 6(a) shows the schematic diagram of a typical holographic memory[41-47] which consists of a laser source, a beam expander and a pinhole spatial fliter, XY beam defiectors, a holographic recording medium, an imaging lens and a 2D detector array. The $X Y$ beam deflectors which consist of two acoustooptic or electrooptic deflectors with anamorphic optical components direct the laser beam to the hologram according to the angle defined by the given address. The light dlffracted from the hologram is imaged onto a $2 \mathrm{D}$ detector array by a lens. A disadvantage of this system is that it is bulky and complicated. Moreover, the capacity-speed product is inherently limited by the frequency bandwidth of the deflectors[42]. For example, an acoustooptic deflector with a frequency bandwidth 
$\Delta f=100 \mathrm{MHz}$ allows only 50 resolvable directions into which the beam can be directed within an access time of $1 \mu \mathrm{sec}$.

The complicated $X Y$ beam deflector can be replaced by a $2 D$ SLM (spatial light modulator) as is shown in Figure 6(b) and has been used in holographic associative memories[22,23]. However, the system utilizing a SLM is still bulky and the reconstructed image quality is normally poor because of the limited resolution and poor contrast of the current SLM'S. Moreover, only a very small portion of the light incident onto a SLM can be used to reconstruct images, leading to extremely low light efficiency.

We have demonstrated a compact and ultra-fast holographic read-only memory[48] that eliminates the need for a bulky laser, SLM's or beam steering optics by using the SELDA in the configuration shown in Figure 6(c). The light from each laser element of the SELDA becomes a tilted plane wave after passing through the lens, $L_{1}$. The tilt angle of the plane wave is determined by the position of the corresponding laser and the focal length of the lens, $L_{1}$. Therefore the SELDA combined with a collimating lens dispenses with the bulky laser and complicated beam steering optics required by other holographic memories. By using this simple and compact optical setup, any frame can be randomly accessed in less than 100 picoseconds ( whout considering detection time ). Also, simultaneous readout of many frames, which would require many sets of $X Y$ deflectors in the system shown in Fig. 6(a), is easily achieved. Furthermore, the quality of the reconstructed images from the system is very high due to the high resolution and contrast of the SELDA.

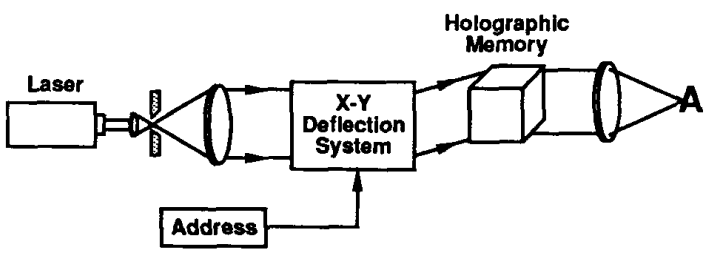

(a)

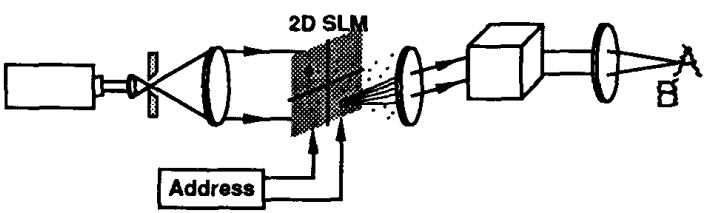

(b)

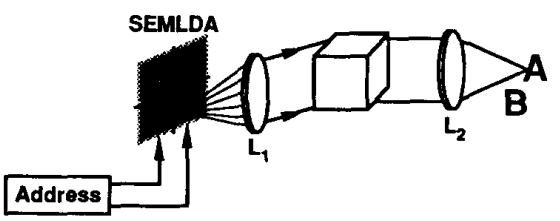

(c)

Fig. 6 Holographic memory readout systems : (a) A typical system using beam deflection systems; (b) a system using a SLM; (c) a compact and ultra-fast memory system using a surface emitting microlaser array.
Most holographic recording medla presently available are not sensitive at the SEL wavelength of $960 \mathrm{~nm}$. However, as described below, the recording of holograms can be performed on a separate system using an Argon laser with wavelength of $514.5 \mathrm{~nm}$. The recording of the holograms is accomplished with a conventional Fourier hologram setup in order to minimize the aberration effects when the hologram is read out with a wavelength different from that used for recording. Most of the aberrations due to the wavelength change are related with the curvature of the wavefront which becomes minimum when the beams are plane waves. Furthermore, this Fourier transform hologram provides distributed information storage of data and high storage capacity. To falthfully record images with high fidelity, a diffuser was placed in front of an input transparency[42]

In the first experiment, a plane hologram was recorded on a TP plate (Thermoplastic Plate, Model No. HC-301, Newport Corp.). The input images, arranged in a 4 by 4 array as shown in Figure 7 (a), are simultaneously recorded by using an Argon laser. The angle between the input and the reference beam in this experiment is 24 degrees at which the efficiency of the TP plate is maximum. The plane holograms thus recorded are reconstructed on the readout system shown in Fig. 6(c). The light from each of the SEL's becomes a tilted plane wave after passing through a lens, $L_{1}$ with the focal length of $10 \mathrm{~cm}$. The size of the SEL used in this readout is $25 \mu \mathrm{m} \times 25 \mu \mathrm{m}$. Figure 7 (c) and (d) show the reconstructed images by the light from the microlasers situated at $(1,4)$ and $(4,2)$ positions, respectively. Although the wavelengths are significantly different between recording and readout, high quality reconstructed images with around $10^{5}$ resolvaible spots are obtained. The reconstructed images were detected by a CCD camera and a monitor, through which the data were obtained. Part of the image degradation is due to the limited resolution of the CCD and the video monitor.

When the memories are recorded on a plane hologram, whole memory sets are reconstructed by a plane wave. The necessary information is retrieved through a window which is located at the origin of output plane, by the positioning relationship $[17,22,23]$. Therefore each laser has to be separated from the adjacent one by each memory size. This limits the capacity of the memory which is given by the SBP (spacebandwidth product) of the recording medium (typically on the order of $\left.10^{8}\right)$. However, it is still quite useful for holographic associative memories due to its shift-invariance property.

In the second experiment, memorles are recorded on a $\mathrm{LiNbO}_{3}$ crystal $\left(0.01 \% \mathrm{Fe}\right.$ doped LiNbO, $20 \times 20 \times 7 \mathrm{~mm}^{3}$, Deltronic Co.) to increase the storage capacity using the third dimension avaliable in a volume storage medium $[41,50]$. The setup to record the volume holograms is the same as for plane holograms. During recording, both reference and object beams are polarized normal to the plane of incidence (ordinary polarization) to reduce spurious scattering effects due to the nonlinear beam coupling[49]. The c-axis of the crystal is oriented along the grating direction as in normal recording. The crystal is rotated by 0.02 degrees between recording holograms to ensure angular separation for selective readout.

For readout, the crystal is oriented to satisfy the phase matching condition at readout wavelength, $960 \mathrm{~nm}$, and is illuminated by a highly collimated beam from a single element of the SELDA to reduce the aberration effect. Fig. 8 shows the reconstructed images from the volume hologram by using the light from SELDA. Each laser is separated from the adjacent one by $70 \mu \mathrm{m}$, corresponding to an angular separation of about 0.04 degrees which corresponds to 0.02 degrees for the recording wavelength. As can be seen in the Figure, the two independent lasers separated by about 0.04 degrees reconstruct 


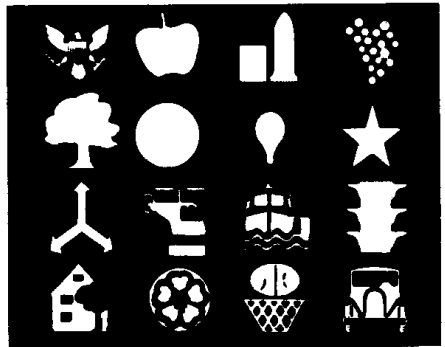

(a)

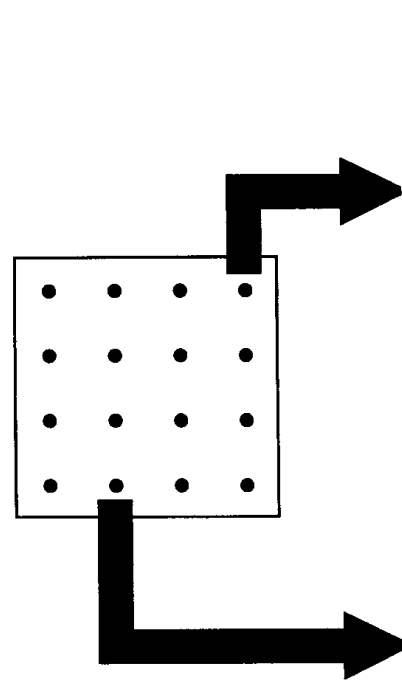

(b)

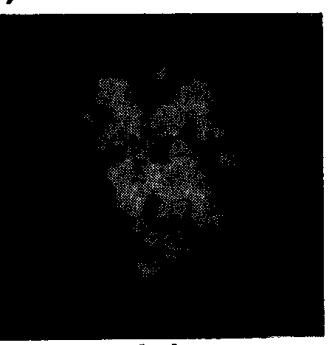

(c)

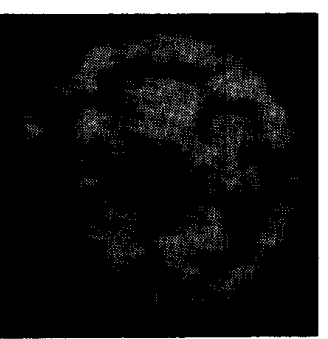

(d)

Fig. 7 Reconstructed images from a plane hologram. (a) Original inputs stored on the holographic memory; (b) SELDA; (c) reconstructed images from microlasers at $(1,4)$ position; (d) and $(4,2)$ position, respectively.

totally different images. In this way, each microlaser can be matched to a separate page, allowing the array to form a selective address generator. To fully utilize the $2 \mathrm{D}$ nature of the SELDA, fractal patterns[50] can be used on the input memories and the SELDA. Although the wavelength changes are signiflcant between recording and reconstruction, faithful highfidelity images can be recalied from the SELDA by recording Fourier holograms.

In the future, SELDA's operating at shorter wavelengths together with new photorefractive materials which allow faster recording and nondestructive readout may make this system architecture extremely practical for fast read-write-erase memory due to its compactness and ultra-fast parallel access capabilities. In this case, the SELDA can work as a page composer as well as a readout system. The system can also be used for other applications such as optical interconnections when the SELDA is incorporated with a detector array.

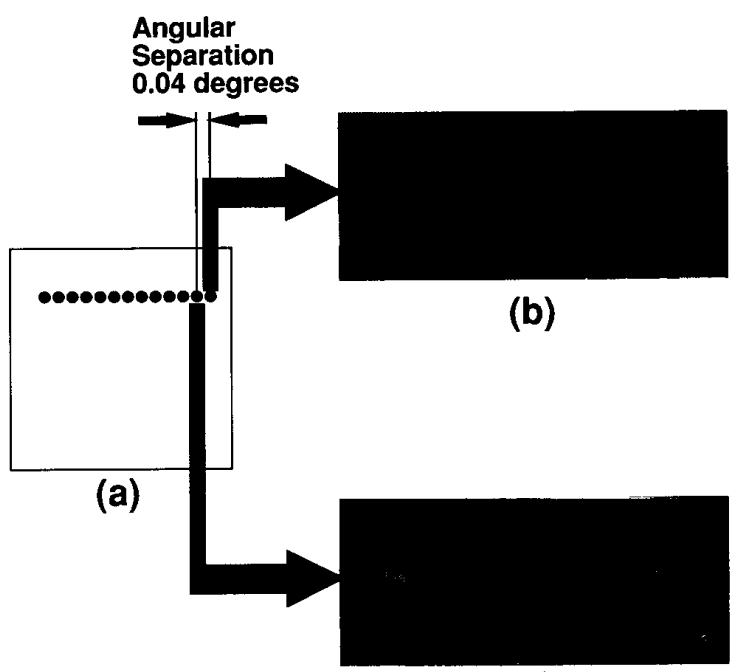

(c)

Fig. 8 Reconstructed images from a volume hologram from the laser 1 (b) and the laser 2 (c). The two independent lasers separated by $70 \mu \mathrm{m}$ (angular separation : 0.04 degrees) reconstruct totally different imaaes.

\section{A COMPACT AND ROBUST INCOHERENT HOLOGRAPHIC CORRELATOR USING A MICRO-LASER DIODE ARRAY}

Next, the recognition part of Fig. 1, which is basically a correlator, will be described. This correlation is the most important computation in artificial neural nets and can be obtained simply by using the VanderLugt correlator. However, the VanderLugt correlator is very sensitive to filter positioning and the transmission quality of the substates of the input and filter. This is because the system relies on the matched filtering where the wavefront distortion of the input signal beam has to be completely compensated by the filter whose trasfer function is given by the complex conjugate of the Fourier tranform of the signal. Therefore, even the slightest misalignment of the filter or the phase distortion of the substrates would affect interference between the reconstructed images generated from many different sources of the input, losing correlation peaks or the shift invariance. If the phase-sensitive interference can be removed, a robust incoherent correlator can be obtained[51].

Although the SELDA cannot work as a SLM because the phases of indlvidual lasers are not locked together, this feature of spatial incoherence can used to average out the interference term. The correlator using the SELDA as an input device is compact because it does not require a bulky laser or a SLM. Moreover, it is not critical to the input and filter making the system very robust, without requiring a heavy optical table.

\section{CONCLUSION}

In conclusion, we have shown that the Vandertugt correlator is powerful in implementing various neural network models for two dimensional images. By using the recently developed surface 
emitting micro-laser diode array, the coherent optical systems can be made compact and robust, still preserving the power of coherent optics.

\section{REFERENCES}

[1] J.J. Hopfield, "Neural Networks and Physical Systems with Emergent Collective Computational Abilities," Proc. Natl. Acad. Sci. U.S.A. 79, pp. 2554-2558, 1982.

[2] F. Rosenblatt, Cornell Aeronaut. Lab. Report, 85-460-1, 1957.

[3] D.E. Rumelhart and J.L. McClelland, Parallel Distributed Processing, Cambridge, MA: M.I.T. (1986).

[4] J.W. Goodman, Introduction to Fourier Optics, McGraw Hill, NY (1968).

[5] A. VanderLugt, "Signal detection by complex spatial filtering," IEEE Trans. Inform. Theory, IT-10, 139, 1964.

[6] N.H. Farhat, D. Psaltis, A. Prata, and Eung Gi Paek, "Optical Implementations of the Hopfield Model," Appl. Opt., 24, 1469, 1985.

[7] D.Z.Anderson, "Coherent Optical Eigenstate Memory," Opt. Lett. 11, 56 (1986).

[8] B.H.Soffer, G.J.Dunning, Y.Owechko, and E.Marom, "Associative Holographic Memory with Feedback Using Phase-Conjugate Mirrors," Opt. Lett. 11118 (1986).

[9] G.H.Dunning, E.Marom, Y.Owechko, and B.H.Soffer, "Optical Holographic Associative Memory Using a Phase Conjugate Resonator," Proc. SPIE, 625, 205 (1986).

[10] A.Yariv, S.Kwong, and K.Kyuma, "Optical Associative Memories Based on Photorefractive Oscillations," Proc. SPIE. 613, 1 (1986).

[11] A.Yariv and S.K.Kwong, "Demonstration of All-optical Associative Holographic Memory," Appl. Phys. Lett. 48, 1114 (1986).

[12] H.K.Liu, S.Y.Kung, and J.A.Davis, "Real-Time Optical Associative Retrieval Technique," Opt. Eng. 25(7), 853 (1986).

[13] A.D.Fisher, C.L.Giles, and J.N.Lee, "Associative Processor Architectures for optical Computing," J. Opt. Soc. Am., A1, 1337 (1984).

[14] R.A.Athale, "Attentive Associative Architectures and Their Implications to Optical Computing," Proc. SPIE., 625, 179 (1986).

[15] C.C.Guest and R.T.Kolste, "Designs and Devices for Optical Bidirectional Associative Memories," Appl. Opt. 26, 5055 (1987).

[16] K.M.Johnson, "Optical Associative Memory Utilizing Electrically and Optically Addressed Liquid Crystal Spatial Light Modulators," OSA Topical Meeting on Optical Computing, Salt lake City, 32 (1989).

[17] Eung Gi Paek and D. Psaltis, "Optical Associative Memory Using Fourier Transform Holograms," Opt. Eng., 26(5), 428, 1987.

[18] Eung Gi Paek and D.Psaltis, "Holographic Implementation of a Neural Network," OSA Annual Meeting. (Seattle, Washington, 1986).

[19] Yuri Owechko, "Nonlinear Holographic Associative Memories," IEEE J. QE, 25 (3), pp. 619-634 (1989).
[20] D. W. Tank and J. J. Hopfield, " Neural Computation by Concentrating Information in Time," Proc. Nat. Acad. Sci., 1896, 1987.

[21] D. W. Tank and J. J. Hopfield, " Concentrating Information in Time : Analogue Neural Networks with Applications to Speech Recognition Problems," Proc. IEEE First Intl. Conf. on Neural Networks, IV, 455, 1987.

[22] Eung $\mathrm{Gi}$ Paek and A. Von Lehmen, "Holographic Associative Memory for Word-break Recognition," Opt. Lett 14, 205, 1989.

[23] Eung Gi Paek and A. Von Lehmen, "Real-Time Holographic Associative Memory for Identifying Words in a Continuous Letter String," Opt. Eng. 28(5), pp. 519-525, 1989

[24] K.Wagner and D.Psaltis, "Multilayer Optical Learning Networks," Appl. Opt. 26, 5061 (1987).

[25] D.Z.Anderson and D.M.Lininger, "Dynamic Optical Interconnects : Volume Holograms as Optical Two-Port Operators," Appl. Opt. 26, 5031 (1987).

[26] Y.Owechko, "Optoelectronic Resonator Neural Networks," Appl. Opt. 26, 5104 (1987).

[27] Y. Owechko and B.H. Soffer, Proc. of IEEE International Conference on Neural Networks, San Diego, CA, June 2427, 1988, II, 385.

[28] Y.Owechko, "Self-Pumped Optical Neural Networks," OSA Topical Meeting on Optical Computing, Salt lake City, 44 (1989).

[29] U.Efron and Y.Owechko, "Optical Implementation of Association and Learning Based on PRIMO/Light Valve Devices," OSA Topical Meeting on Optical Computing, Salt lake City, 311 (1989)

[30] N.H.Farhat, "Optoelectronic Analogs of Self-Programming Neural Nets : Architecture and Methodologies for Implementing Fast Stochastic Learning by Simulated Annealing," Appl. Opt. 26, 5093 (1987).

[31] M. Lemmon and B.V.K. Vijaya Kumar, "Competitively Inhibited Optical Neural Networks Using Two-Step Holographic Materials," OSA Topical Meeting on Optical Computing, Salt lake City, 36 (1989).

[32] M. Ishkawa, N. Mukohzaka, H. Toyoda, and Y. Suzuki, Appl. Opt. 28, 291 (1988).

[33] A.D.Fisher, W.L.Lippincott, and J.N.Lee, "Optical Implementations of Associative Networks with Versatile Adaptive Learning Capabilities," Appl. Opt. 26, 5039 (1987).

[34] D. Psaltis, D. Brady, and K. Wagner, "Adaptive Optical Networks Using Photorefractive Crystals," Appl. Opt. 9, 1752 (1988).

[35] N.H. Farhat and Z.Y. Shae, "Bimodal Stochastic Optical Learning Machine," Proc. of IEEE International Conference on Neural Networks, San Diego, CA, II, 365 (1988).

[36] J.H.Hong and Pochi Yeh, OSA Topical Meeting on Optical Computing, Salt lake City, 307 (1989).

[37] Ken-ichi Kitayama, Hisao Yoshinaga, and Tsutomu Hara "Experiments of Learning in Optical Perceptron-like and Multilayer Neural Networks," Proc. of IJCNN, Washington D.C. II -465 (1989).

[38] R.O. Duda and P.E. Hart, Pattern Classification and Scene Analysis, Wiley-Int. NY (1973).

[39] Eung Gi Paek, J.R. Wullert II and J.S. Patel, "Holographic Learning Machine for Multi-category Classification," to be 
published in Opt. Lett.

[40] J.Jewell, A.Scherer, S.L.McCall, Y.H.Lee, J.P.Harbison, and L.T.Florez, Electronics Lett. (To be published.)

[41] P.J. van Heerden, "Theory of Optical Information Storage in Solids," Appl. Opt. 2, 393 (1963).

[42] R.J.Collier, C.B.Burckhart, and L.H.Lin, Optical Holography (Academic Press, New York) (1971).

[43] Caulfield, Holography Handbook, Academic Press (1979).

[44] Ld'Auria, J.P.Huignard, C.Slezak, and E.Spitz, "Experimental Holographic Read-Write Memory Using 3-D Storage," Appl. Opt., 13, 808 (1974).

[45] D.Chen and J.D.Zook, "An Overview of Optical Storage Technology," Proc. IEEE, 63, 1207 (1975).

[46] D.Psaltis, M.A.Neifield, and A.Yamamura, "Image Correlators Using Optical Memory Disks," Opt. Lett., 14, 429 (1989).

[47] S. Redfield and L Hesselink, "Enhanced Nondestructive Holographic Readout in Strontium Barium Niobate," Opt. Lett., 13, 880 (1989).

[48] Eung Gi Paek, John R.Wullert II, M.Jain, A.Von Lehmen, A.Scherer, J.Harbison, H.J.Yu, and R.Martin, "A Compact and Ultra-Fast Holographic Memory Using a Surface Emitting Micro-Laser Diode Array," submitted to Opt. Lett.

[49] R. Magnusson and T.K. Gaylord, "Use of Dynamic Theory to Describe Experimental Results from Volume Holography," J. Appl. Phys. 47, 190 (1976).

[50] D.Psaltis, J.Yu, X.G.Gu, and H.Lee, Tech. Digest, Topical Meeting Opt. Compt., Lake Tahoe, pp. 129-132 (1987).

[51] A.W. Lohmann, "Matched Filtering with Self-Luminous Objects," Appl. Opt. 7, 561 (1968). 